\title{
Least Squares Estimator for Vasicek Model Driven by Fractional Lévy Processes
}

\author{
QingBo Wang, Xiuwei Yin
}

School of mathematics and statistic, Anhui Normal University, Wuhu 241000, P.R. China

qbwang3@163.com, xweiyin@163.com

\section{Abstract}

In this paper, we consider parameter estimation problem for Vasicek model driven by fractional lévy processes defined

$$
d X_{t}=\left(\mu-\theta X_{t}\right) d t+d L_{t}^{d}, t \geq 0, X_{0}=0 .
$$

We construct least squares estimator for drift parameters based on time-continuous observations, the consistency and asymptotic distribution of these estimators are studied in the non-ergodic case. In contrast to the fractional Vasicek model, it can be regarded as a Lévy generalization of fractional Vasicek model.

Keywords: Stochastic differential equations, fraction Levy processes, least squares estimator, asymptotic distribution.

Subject Classification: 60G18, 60G22, 65C30, $93 \mathrm{E} 24$.

Date of Publication: 2018-11-01

ISSN: 2347-1921

Volume: 14 Issue: 02

Journal: JOURNAL OF ADVANCES IN MATHEMATICS

Website: https://cirworld.com

This work is licensed under a Creative Commons Attribution 4.0 International License

How to Cite: wang, qingbo, \& Yin, X. (2018). Least Squares Estimator for Vasicek Model Driven by Fractional Levy Processes. JOURNAL OF ADVANCES IN MATHEMATICS, 14(2), 8013-8024. Retrieved from https://cirworld.com/index.php/jam/article/view/7839 


\section{Introduction}

Statistical inference for stochastic differential equations is a main research direction in probability theory and its applications. When the noise is a standard Brownian motion, such problems have been extensively studied and some surveys and complete literatures for this direction could be found in Bishwal [2]. Moreover, since the seminal work of Vasicek [15], the Vasicek model

$$
d X_{t}=\left(\mu-\theta X_{t}\right) d t+d B_{t}, \quad t \geq 0, \quad X_{0}=0,
$$

driven by standard Brownian motion $\left(B_{t}\right)_{t \geq 0}$ has been extensively applied in various fields, such as economics and finance, biology, physics, chemistry, medicine and environmental studies, where $\mu, \theta$ are unknown, the first term describes the so-called drift component $\left(\mu-\theta X_{t}\right) d t$. The parameter $\theta$ determines the reversion speed of the stochastic component to their long-term mean $\frac{\mu}{\theta}$. The economic interpretation of this mean-reversion component is that stochastic price fluctuations around the mean and price peaks are only temporarily, caused by for example power plant outages or capacity shortages. Indeed, when this model is used to describe some phenomena, it is important to identify the unknown parameters in this model. As a result, the parameter estimation problem for the Vasicek process driven by Brownian motion has played an important role in econometrics and becomes a interesting problem in the literature.

When $\mu=0$, the process degenerates into the well-known Ornstein-Uhlenbeck process. If the parameter $\theta \in(-\infty,+\infty)$ is unknown and the process $\left\{X_{t}, t \geq 0\right\}$ can be observed continuously, then an important problem is to estimate the parameter $\theta$ based on the (single path) observation $\left\{X_{t}, t \geq 0\right\}$. The most popular approaches are either the maximum likelihood estimators (MLE) or the least squares estimators (LSE), and in this case they coincide. For $\theta>0$ (ergodic case), the MLE of $\theta$ is asymptotically normal (Kutoyants [9]). For $\theta<0$ (non-ergodic case), the MLE of $\theta$ is asymptotically Cauchy (Dietz and Kutoyants [6]).

As an extension of Brownian motion, the fractional Brownian motion ( $\mathrm{fBm}$ ) has become an object of intensive study, due to its interesting properties and its applications in various scientific areas such as hydrology, telecommunications, fluid dynamics, turbulence, image processing, economics and finance. Recall that the $\mathrm{fBm}$ $\left\{B_{H}(t)\right\}_{t \geq 0}$ with Hurst index $H \in(0,1)$ is the only centred Gaussian self-similar process with stationary increments, satisfies $B_{0}^{H}=0, E\left[B_{t}^{H}\right]=0, t \geq 0$, the covariance function is given by

$$
E\left[B_{t}^{H} B_{s}^{H}\right]=\frac{1}{2}\left[t^{2 H}+s^{2 H}-|t-s|^{2 H}\right], \quad t, s \geq 0 .
$$

It has stochastic integral representation in terms of a standard Brownian motion:

$$
B_{t}^{H}=\frac{\sqrt{2 H \Gamma\left(\frac{3}{2}-H\right)}}{\Gamma\left(H+\frac{1}{2}\right) \Gamma(2-2 H)} \int_{\mathbb{R}}\left[(t-s)_{+}^{H-\frac{1}{2}}-(-s)_{+}^{H-\frac{1}{2}}\right] d B_{s},
$$

where $u_{+}=\max \{u, 0\}, B_{t}$ is standard Brownian motion. For $H=\frac{1}{2}, B^{H}$ coincides with the standard Brownian motion $B$, but $B^{H}$ is neither a semi martingale nor a Markov process unless $H=\frac{1}{2}$.

If the Brownian motion in the Vasicek model (1.1) is replaced with $\mathrm{fBm}$, we get the following fractional Vasicek model ( $\mathrm{fVm}$ )

$$
d X_{t}=\left(\mu-\theta X_{t}\right) d t+d B_{t}^{H}, \quad t \geq 0, \quad X_{0}=0 .
$$

Parameter $\theta$ determines the persistence in $X_{t}$. Depending on the sign of $\theta$, the model can capture the stationary, the explosive, and the null recurrent behavior. The $\mathrm{fVm}$ was first used to describe the dynamics in volatility by Comte and Renault [3]. Other applications of $\mathrm{fVm}$ can be found in Comte, Coutin and Renault [4], Corlay, Lebovits 
and Véhel [5] references therein. Despite many applications of $\mathrm{fVm}$ in practice, estimation and the asymptotic theory in $\mathrm{fVm}$ has received little attention in the literature. Xiao and $\mathrm{Yu}$ [16] propose estimators for $\mu$ and $\theta$ develop the asymptotic theory for the estimators.

When $\mu=0$, a very important special case of $\mathrm{fVm}$ is the fractional Ornstein-Uhlenbeck process. The parameter estimation for $\theta$ has been extensively studied using the MLE method (see Prakasa Rao [11]) or using the LSE technique (see Hu and Nualart [8]).

On the basis of sufficient study of $\mathrm{fBm}$, many authors have proposed to use more general stochastic processes and random fields as stochastic models. Such applications have raised many interesting theoretical questions about stochastic processes and fields in general. Therefore, some generalizations of the $\mathrm{fBm}$ have been introduced such as sub-fractional Brownian motion, bifractional Brownian motion, weighted-fractional Brownian motion, fractional Lévy processes. However, in contrast to the extensive studies on $\mathrm{fBm}$, there has been only a little systematic investigation on the statistical inference of other fractional processes. The main reason for this is the complexity of dependence structures. Recently, Fink and Klüppelberg [7] proved that the fractional Lévy driven Ornstein-Uhlenbeck processes (FLOUP) has unique stationary pathwise solution of the corresponding Langevin equation and the increments of an FLOUP exhibits long-range dependence.

However, there has been no study on parametric inference for Vasicek model with fractional Lévy noises yet. Motivated by the aforementioned works, as a first attempt, in this paper, we consider the generalized Vasicek model driven by fractional Lévy process $L_{t}^{d}$ (the precise definition is given below in Definition 2.1), and it is defined by the following stochastic differential equations

$$
d X_{t}=\left(\mu-\theta X_{t}\right) d t+d L_{t}^{d}, \quad t \geq 0, \quad X_{0}=0 .
$$

In the present paper, we assume that the parameters $\mu \in R$ and $\theta<0$ are unknown. We shall use the least square method to construct their estimators under the continuous observations, respectively. Our main results and aims are described as follows.

Firstly, we use the least square method to obtain the estimators of $\mu$ and $\theta$. We introduce least squares estimators of $\mu$ and $\theta$ of the forms

$$
\hat{\theta}_{T}=\theta-\frac{T \int_{0}^{T} X_{t} d L_{t}^{d}-L_{T}^{d} \int_{0}^{T} X_{t} d t}{T \int_{0}^{T} X_{t}^{2} d t-\left(\int_{0}^{T} X_{t} d t\right)^{2}},
$$

and

$$
\hat{\mu}_{T}=\frac{1}{T}\left(X_{T}+\hat{\theta}_{T} \int_{0}^{T} X_{t} d t\right)
$$

for all $T>0$. The two estimators are motivated by the following heuristic argument. By minimizing the contrast function

$$
L(\theta, \mu)=\int_{0}^{T}\left|\dot{X}_{t}-\left(\mu-\theta X_{t}\right)\right|^{2} d t
$$

where $\dot{X}_{t}$ denotes the differentiation of $X_{t}$ with respect to $t$.

As a result, we can explicitly get the two least squares estimators $\hat{\theta}_{T}$ and $\hat{\mu}_{T}$ as follows 


$$
\begin{gathered}
\hat{\theta}_{T}=\frac{X_{T} \int_{0}^{T} X_{t} d t-T \int_{0}^{T} X_{t} d X_{t}}{T \int_{0}^{T} X_{t}^{2} d t-\left(\int_{0}^{T} X_{t} d t\right)^{2}}, \\
\hat{\mu}_{T}=\frac{X_{T} \int_{0}^{T} X_{t}^{2} d t-\int_{0}^{T} X_{t} d X_{t} \int_{0}^{T} X_{t} d t}{T \int_{0}^{T} X_{t}^{2} d t-\left(\int_{0}^{T} X_{t} d t\right)^{2}},
\end{gathered}
$$

for all $T>0$, where the integral $\int_{0}^{T} X_{t} d X_{t}$ is interpreted as the Young integral (see, for example, Young [10]). We shall prove the consistency of $\hat{\theta}_{T}$ and $\hat{\mu}_{T}$, that is,

$$
\hat{\theta}_{T} \stackrel{a . s .}{\longrightarrow} \theta, \quad T \rightarrow \infty
$$

and

$$
\hat{\mu}_{T} \stackrel{a . s .}{\longrightarrow} \mu, \quad T \rightarrow \infty
$$

where the notation $\stackrel{\text { a.s. }}{\longrightarrow}$ denotes "almost surely convergence".

The rest of this paper is organized as follows. In Section2, we present some preliminaries for lévy process and fractional lévy process. In Section3, we study the consistency of the least square's estimator $\hat{\theta}_{T}$ and $\hat{\mu}_{T}$.

\section{Preliminaries}

2.1. Lévy processes. In this subsection, we mainly introduce the elementary properties of Lévy processes that will be used in following. More studies on the Lévy process can be found in Sato [13], Samorodnitsky and Taqqu [12] and the references therein.

Let $L=\{L(t)\}_{t \geq 0}$ be Lévy processes in $\mathbb{R}$ without Brownian component. It is determined by its characteristic function in the Lévy-Khintchine form

$$
\mathbb{E}[\exp \{i u L(t)\}]=\exp \{t \psi(u)\}, t \geq 0,
$$

where

$$
\psi(u)=i \gamma u+\int_{\mathbb{R}}\left(e^{i u x}-1-i u x 1_{|x| \leq 1}\right) \nu(d x), u \in \mathbb{R},
$$

where $\gamma \in \mathbb{R}$ and $\nu$ is the Lévy measure of $L$ on $\mathbb{R}$ that satisfies

$$
\nu(\{0\})=0, \quad \int_{\mathbb{R}}\left(|x|^{2} \wedge 1\right) \nu(d x)<\infty, \quad \int_{|x|>1}|x|^{2} \nu(d x)<\infty .
$$

This is a necessary and sufficient condition for $L$ to have finite mean and variance given by

$$
\operatorname{var}(L(t))=t \operatorname{var}(L(1))=t \int_{\mathbb{R}} x^{2} \nu(d x), t \geq 0
$$

Furthermore, we restrict $E[L(1)]=0$, then 


$$
\gamma=-\int_{|x|>1} x \nu(d x)
$$

and

$$
\psi(u)=\int_{\mathbb{R}}\left(e^{i u x}-1-i u x\right) \nu(d x), u \in \mathbb{R}
$$

Throughout this paper we will use a two side Lévy process $L=\{L(t)\}_{t \in R}$ constructed by taking two independent copies $\left\{L_{1}(t)\right\}_{t \geq 0},\left\{L_{2}(t)\right\}_{t \geq 0}$ of a one-side Lévy process and setting

$$
L(t)=\left\{\begin{array}{cc}
L_{1}(t), & t \geq 0 \\
-L_{2}\left(-t_{-}\right), & t<0
\end{array}\right.
$$

2.2 Fractional Lévy processes. In this subsection, we briefly recall the definition and properties of fractional Lévy process.

As an extension of fractional Brownian motion, fractional Lévy process is of interest in practical applications because of its stationarity of increments and long-range dependence. However, it is not Gaussian. Actually, the very large utilization of the fractional Brownian motion in practice (hydrology, telecommunications) are due to these properties (long range dependence). One prefers in general fractional Brownian motion before other processes because it is Gaussian and the calculus for it is easier. However, in concrete situations when the Gaussianity is not plausible for the model, one can use for example the fractional Lévy process. There exists a consistent literature that focuses on different theoretical and applications aspects of the fractional Lévy process. For example, Bender, Lindner and Schick'[1] studied the finite variation of fractional Lévy processes, Tikanmäki and Mishura [14] define fractional Lévy processes using the compact interval representation and proved that the fractional Lévy processes presented via different integral transformations have the same finite dimensional distributions if and only if they are fractional Brownian motions.

In this paper, we are interested in fractionally integrated processes. Therefore, we will work with the fractional integration parameter $d=H-\frac{1}{2} \in\left(-\frac{1}{2}, \frac{1}{2}\right)$ rather than the Hurst parameter $H$. Moreover, we restrict ourselves to $d \in\left(0, \frac{1}{2}\right)$ as we are interested in the long range dependence case. Based on the moving average integral representation of fractional Brownian motion, the class of fractional Lévy processes is introduced by replacing the Brownian motion by a general Lévy process with zero mean, finite variance and no Brownian component.

Definition 2.1. (Marquardt [10]) Let $L=\left(L_{t}\right)_{t \in R}$ be a zero-mean two-sided Lévy process with $E\left[L(1)^{2}\right]<\infty$ and without a Brownian component. For fractional integration parameter $d \in\left(0, \frac{1}{2}\right)$, a stochastic process

$$
L_{t}^{d}:=\frac{1}{\Gamma(d+1)} \int_{\infty}^{\infty}\left[(t-s)_{+}^{d}-(-s)_{+}^{d}\right] L(d s), \quad t \in R
$$

is called a fractional Lévy process ( $f$ Lp).

fLp has the sample path properties as follows.

- Hölder continuity. For every $\beta<d$, there exists a continuous modification of $L^{d}$ and there exist an almost surely positive random variable $H_{\epsilon}$ and a constant $\delta>0$ such that

$$
P\left[\omega \in \Omega: \sup _{0<h<H_{\epsilon}(\omega)}\left(\frac{L_{t+h}^{d}(\omega)-L_{t}^{d}(\omega)}{h^{\beta}}\right) \leq \delta\right]=1 .
$$


- Stationary increments. $L^{d}$ is a process with stationary increments.

- Symmetry. $\left\{L_{-t}^{d}\right\}_{t \in \mathbb{R}} \stackrel{d}{=}\left\{-L_{t}^{d}\right\}_{t \in \mathbb{R}}$.

$L^{d}$ is locally self-similar with parameter $\widetilde{H}$, that is, for every fixed $t \in R$,

$$
\lim _{\epsilon \rightarrow 0}\left\{\frac{L_{t+\epsilon x}^{d}-L_{t}^{d}}{\epsilon^{\widetilde{H}}}\right\} \stackrel{d}{=}\left\{Y_{\widetilde{H}}(x)\right\}, \quad x \in \mathbb{R},
$$

here $Y_{\widetilde{H}}$ is a linear fractional stable motion with expression

$$
Y_{\widetilde{H}}(t)=\frac{1}{\Gamma(d)} \int_{\mathbb{R}}\left[(t-s)_{+}^{\widetilde{H}-\frac{1}{\alpha}}-(-s)_{+}^{\widetilde{H}-\frac{1}{\alpha}}\right] L_{\alpha}(d s),
$$

where $L_{\alpha}$ is symmetric $\alpha$-stable Lévy process (see Samorodnitsky and Taqqu[12]).

The following two Lemma gives an integral relationship between fractional lévy processes and integral with Lévy processes and the second-order property of the stochastic integral respect to fractional lévy processes.

Lemma2.1. (Marquardt[10]) Let $g \in H, H$ is the completion of $L^{1}(R) \cap L^{2}(R)$ with respect to the norm $\|g\|_{H}^{2}=E\left[L(1)^{2}\right] \int_{R}\left(I_{-}^{d} g\right)^{2}(u) d u$, then

$$
\int_{R} g(s) d L_{s}^{d}=\int_{R}\left(I_{-}^{d} g\right)(u) d L_{u}
$$

where the equality holds in the $L^{2}$ sense and $I_{-}^{d} g$ denotes the Riemann-Liouville fractional integrals defined by

$$
\left(I_{-}^{d} g\right)(x)=\frac{1}{\Gamma(d)} \int_{x}^{\infty} g(t)(t-x)^{d-1} d t
$$

Lemma2.2. (Marquardt[10]) Let $|f|,|g| \in H$. The

$$
\begin{aligned}
& E\left[\int_{R} g(s) d L_{s}^{d} \int_{R} f(s) d L_{t}^{d}\right]=\frac{\Gamma(1-2 d) E\left[L(1)^{2}\right]}{\Gamma(d) \Gamma(1-d)} \int_{R} \int_{R} f(t) g(s)|t-s|^{2 d-1} d s d t . \\
& \varphi\left(h_{t}, g_{t}\right)=\varphi\left(h_{0}, g_{0}\right)+\int_{0}^{t} \frac{\partial \varphi}{\partial h}\left(h_{u}, g_{u}\right) d h_{u}+\int_{0}^{t} \frac{\partial \varphi}{\partial g}\left(f_{u}, g_{u}\right) d g_{u}, \quad 0 \leq t \leq T .
\end{aligned}
$$

In particular, using the Young integral of (2.4), we can rewrite the solution of (1.5) as

$$
X_{t}=\frac{\mu}{\theta}\left(1-e^{-\theta t}\right)+e^{-\theta t} \int_{0}^{t} e^{\theta u} d L_{u}^{d} .
$$

Furthermore, we have 


$$
\begin{aligned}
\int_{0}^{T} X_{t} d t & =\int_{0}^{T}\left[\frac{\mu}{\theta}\left(1-e^{-\theta t}\right)+e^{-\theta t} \int_{0}^{t} e^{\theta u} d L_{u}^{d}\right] d t \\
& =\frac{\mu}{\theta}\left(T+\frac{e^{-\theta T}-1}{\theta}\right)+\int_{0}^{T} \int_{0}^{t} e^{-\theta t} e^{\theta u} d L_{u}^{d} d t \\
& =\frac{\mu}{\theta}\left(T+\frac{e^{-\theta T}-1}{\theta}\right)+\int_{0}^{T} \int_{u}^{T} e^{-\theta t} d t e^{\theta u} d L_{u}^{d} \\
& =\frac{1}{\theta}\left(\mu T-\frac{\mu}{\theta}+L_{T}^{d}\right)+e^{-\theta T}\left[\frac{\mu}{\theta^{2}}-\frac{\int_{0}^{T} e^{\theta u} d L_{u}^{d}}{\theta}\right]
\end{aligned}
$$

According to (1.9), (1.10) and (2.5), we can rewrite $\hat{\theta}_{T}$ and $\hat{\mu}_{T}$ as

$$
\begin{aligned}
\hat{\theta}_{T} & =\frac{X_{T} \int_{0}^{T} X_{t} d t-T \int_{0}^{T} X_{t}\left[\left(\mu-\theta X_{t}\right) d t+d L_{t}^{d}\right]}{T \int_{0}^{T} X_{t}^{2} d t-\left(\int_{0}^{T} X_{t} d t\right)^{2}} \\
& =\frac{\theta T \int_{0}^{T} X_{t}^{2} d t-T \int_{0}^{T} X_{t} d L_{t}^{d}-\left(\theta \int_{0}^{T} X_{t} d t-L_{T}^{d}\right) \int_{0}^{T} X_{t} d t}{T \int_{0}^{T} X_{t}^{2} d t-\left(\int_{0}^{T} X_{t} d t\right)^{2}} \\
& =\theta+\frac{L_{T}^{d} \int_{0}^{T} X_{t} d t-T \int_{0}^{T} X_{t} d L_{t}^{d}}{T \int_{0}^{T} X_{t}^{2} d t-\left(\int_{0}^{T} X_{t} d t\right)^{2}}
\end{aligned}
$$

and

$$
\hat{\mu}_{T}=\mu+\frac{L_{T}^{d} \int_{0}^{T} X_{t}^{2} d t-\int_{0}^{T} X_{t} d t \int_{0}^{T} X_{t} d L_{t}^{d}}{T \int_{0}^{T} X_{t}^{2} d t-\left(\int_{0}^{T} X_{t} d t\right)^{2}}
$$

\section{Asymptotic behavior of the least squares estimator}

In this section, let $\theta<0$, we consider the strong consistency of $\hat{\theta}$ and $\hat{\mu}$. Moreover, we also investigate the asymptotic of the estimator for the long term mean.

Theorem 3.1. Assume $d \in\left(0, \frac{1}{2}\right), \theta<0$. The

$$
\hat{\theta}_{T} \stackrel{a . s .}{\longrightarrow} \theta, \quad T \rightarrow \infty
$$

and

$$
\hat{\mu}_{T} \stackrel{a . s .}{\longrightarrow} \mu, \quad T \rightarrow \infty
$$

For prove Theorem 3.1, we need the following lemma.

Assume $d \in\left(0, \frac{1}{2}\right), \theta<0$. Let $\varphi_{T}$ be defined as $\varphi_{T}=\int_{0}^{T} e^{\theta s} d L_{s}^{d}$. Then $\varphi_{\infty}=\int_{0}^{\infty} e^{\theta s} d L_{s}^{d}$ is welldefined, and as $T \rightarrow \infty$

$$
\varphi_{T} \rightarrow \varphi_{\infty}, \quad \text { almost surely. }
$$

Proof. Using the Young integral and definition of $\varphi_{T}$, we can write 


$$
\varphi_{T}=e^{\theta T} L_{T}^{d}-\theta \int_{0}^{T} L_{s}^{d} e^{\theta s} d s
$$

Hence $\varphi_{T}$ is well-defined, since

$$
\int_{0}^{\infty} e^{\theta s} E\left|L_{s}^{d}\right| d s \leq \sqrt{c} \int_{0}^{\infty} s^{d+\frac{1}{2}} e^{\theta s}<\infty .
$$

Moreover, for any $\varepsilon>0$,

$$
\begin{aligned}
& \sum_{n \geq 0} P\left(\sup _{n \leq t \leq n+1}\left|\int_{t}^{\infty} e^{\theta s} L_{s}^{d} d s\right| \geq \varepsilon\right) \\
& \leq \varepsilon^{-1} \sum_{n \geq 0} E\left(\sup _{n \leq t \leq n+1}\left|\int_{t}^{\infty} e^{\theta s} L_{s}^{d} d s\right|\right) \\
& \leq \varepsilon^{-1} \sum_{n \geq 0} E\left(\left|\int_{n}^{\infty} e^{\theta s}\right| L_{s}^{d}|d s|\right) \\
& \leq \varepsilon^{-1} \sqrt{c} \sum_{n \geq 0} \int_{0}^{\infty} e^{\theta s} s^{d+\frac{1}{2}} d s \\
& \leq \varepsilon^{-1} \sqrt{c} \sum_{n \geq 0} e^{\frac{\theta}{2} n} \int_{0}^{\infty} e^{\frac{\theta}{2} s} s^{d+\frac{1}{2}} d s \\
&=\varepsilon^{-1} \sqrt{c} \Gamma\left(d+\frac{2}{3}\right)\left(\frac{2}{\theta}\right)^{d+\frac{2}{3}} \sum_{n \geq 0} e^{\frac{\theta}{2} n}<\infty .
\end{aligned}
$$

By using Borel-Cantell's lemma, we can obtain $\varphi_{T} \stackrel{\text { a.s. }}{\longrightarrow} \varphi_{\infty}$, as $T \rightarrow \infty$. This completes the proof.

Proof of Theorm 3.1 . By (2.7), we have

$$
\hat{\theta}_{T}-\theta=\frac{L_{T}^{d} \int_{0}^{T} X_{t} d t-T \int_{0}^{T} X_{t} d L_{t}^{d}}{T \int_{0}^{T} X_{t}^{2} d t-\left(\int_{0}^{T} X_{t} d t\right)^{2}}=\frac{\frac{L_{T}^{d} \int_{0}^{T} X_{t} d t-T \int_{0}^{T} X_{t} d L_{t}^{d}}{T e^{-2 \theta T}}}{\frac{T \int_{0}^{T} X_{t}^{2} d t-\left(\int_{0}^{T} X_{t} d t\right)^{2}}{T e^{-2 \theta T}}}
$$

We first consider the term

$$
\frac{T \int_{0}^{T} X_{t}^{2} d t-\left(\int_{0}^{T} X_{t} d t\right)^{2}}{T e^{-2 \theta T}}
$$

From (2.6) and L'Hôspital's rule, we can get

$$
\lim _{T \rightarrow \infty} \int_{0}^{T} e^{\theta T} X_{t} d t=\frac{\mu-\theta \varphi_{\infty}}{\theta^{2}}
$$

And

$$
\lim _{T \rightarrow \infty} \int_{0}^{T} e^{2 \theta T} X_{t}^{2} d t=-\frac{\left(\mu-\theta \varphi_{\infty}\right)^{2}}{2 \theta^{3}}
$$


Combining (3.5) and (3.6), we have

$$
\lim _{T \rightarrow \infty} \frac{T \int_{0}^{T} X_{t}^{2} d t-\left(\int_{0}^{T} X_{t} d t\right)^{2}}{T e^{-2 \theta T}}=-\frac{\left(\mu-\theta \varphi_{\infty}\right)^{2}}{2 \theta^{3}} .
$$

Next we consider term

$$
\frac{L_{T}^{d} \int_{0}^{T} X_{t} d t-T \int_{0}^{T} X_{t} d L_{t}^{d}}{T e^{-2 \theta T}}
$$

From (3.5), we get

$$
\lim _{T \rightarrow \infty} \frac{L_{T}^{d} \int_{0}^{T} X_{t} d t}{T e^{-2 \theta T}}=0 .
$$

By Young integral together with L'Hôspital's rule, we get

$$
\begin{aligned}
\lim _{T \rightarrow \infty} \frac{T \int_{0}^{T} X_{t} d L_{t}^{d}}{T e^{-2 \theta T}} & =\lim _{T \rightarrow \infty} \frac{\int_{0}^{T}\left[\frac{\mu}{\theta}\left(1-e^{-\theta t}\right)+e^{-\theta t} \int_{0}^{t} e^{\theta u} d L_{u}^{d}\right] d L_{t}^{d}}{e^{-2 \theta T}} \\
& =\lim _{T \rightarrow \infty} \frac{\frac{\mu}{\theta} L_{T}^{d}-\frac{\mu}{\theta} \int_{0}^{T} e^{-\theta t} d L_{t}^{d}+\int_{0}^{T} e^{-\theta t} \int_{0}^{t} e^{\theta u} d L_{u}^{d} d L_{t}^{d}}{e^{-2 \theta T}} \\
& =-\frac{\mu}{\theta} \lim _{T \rightarrow \infty} \frac{\int_{0}^{T} e^{-\theta t} d L_{t}^{d}}{e^{-2 \theta T}}+\lim _{T \rightarrow \infty} \frac{\int_{0}^{T} e^{-\theta t} \int_{0}^{t} e^{\theta u} d L_{u}^{d} d L_{t}^{d}}{e^{-2 \theta T}} \\
& =-\frac{\mu}{\theta} \lim _{T \rightarrow \infty} \frac{e^{-\theta T} L_{T}^{d}+\theta \int_{0}^{T} L_{t}^{d} e^{-\theta t} d t}{e^{-2 \theta T}}+\lim _{T \rightarrow \infty} \frac{\int_{0}^{T} e^{-\theta t} \varphi_{t} d L_{t}^{d}}{e^{-2 \theta T}} \\
& =\lim _{T \rightarrow \infty} \frac{\frac{1}{2} e^{-2 \theta T} \varphi_{T}^{2}+\theta \int_{0}^{T} \varphi_{T}^{2} e^{-2 \theta T} d t}{e^{-2 \theta T}} \\
& =\frac{\varphi_{\infty}^{2}}{2}-\lim _{T \rightarrow \infty} \frac{\theta \int_{0}^{T} \varphi_{T}^{2} e^{-2 \theta T} d t}{e^{-2 \theta T}} \\
& =\frac{\varphi_{\infty}^{2}}{2}-\frac{\varphi_{\infty}^{2}}{2}=0 .
\end{aligned}
$$

So, we can easy get

$$
\lim _{T \rightarrow \infty} \frac{L_{T}^{d} \int_{0}^{T} X_{t} d t-T \int_{0}^{T} X_{t} d L_{t}^{d}}{T e^{-2 \theta T}}=0 .
$$

Combining (3.7) and (3.8), we obtain

$$
\hat{\theta}_{T} \stackrel{a . s .}{\longrightarrow} \theta, \quad T \rightarrow \infty .
$$

Next, using the similar method as above, from (2.8), we have

$$
\hat{\mu}_{T}-\mu=\frac{L_{T}^{d} \int_{0}^{T} X_{t}^{2} d t-\int_{0}^{T} X_{t} d t \int_{0}^{T} X_{t} d L_{t}^{d}}{T \int_{0}^{T} X_{t}^{2} d t-\left(\int_{0}^{T} X_{t} d t\right)^{2}} .
$$

Moreover, we can easily obtain 


$$
\lim _{T \rightarrow \infty} \frac{L_{T}^{d} \int_{0}^{T} X_{t}^{2} d t-\int_{0}^{T} X_{t} d t \int_{0}^{T} X_{t} d L_{t}^{d}}{T e^{-2 \theta T}}=0
$$

So,

$$
\hat{\mu}_{T} \stackrel{a . s .}{\longrightarrow} \mu, \quad T \rightarrow \infty .
$$

This completes the proof.

It is easy to see that the long term mean of $X_{t}$ is $\frac{\mu}{\theta}$. It follows from (1.9) and (1.10), we have

$$
\frac{\hat{\mu}_{T}}{\hat{\theta}_{T}}=\frac{X_{T} \int_{0}^{T} X_{t}^{2} d t-\int_{0}^{T} X_{t} d X_{t} \int_{0}^{T} X_{t} d t}{X_{T} \int_{0}^{T} X_{t} d t-T \int_{0}^{T} X_{t} d X_{t}} .
$$

Corollary 3.1. Assume $d \in\left(0, \frac{1}{2}\right), \theta<0$. The

$$
\frac{\hat{\mu}_{T}}{\hat{\theta}_{T}} \stackrel{\text { a.s. }}{\longrightarrow} \frac{\mu}{\theta}, \quad T \rightarrow \infty
$$

By the Young integral, we have

$$
\frac{\hat{\mu}_{T}}{\hat{\theta}_{T}}=\frac{\frac{e^{\theta T}}{T} \int_{0}^{T} X_{t}^{2} d t-\frac{X_{T}}{2 T} e^{\theta T} \int_{0}^{T} X_{t} d t}{\frac{e^{\theta T}}{T} \int_{0}^{T} X_{t} d t-\frac{X_{T}}{2} e^{\theta T}} .
$$

It follows from (2.5) and (3.5), we obtain

$$
\begin{aligned}
\lim _{T \rightarrow \infty}\left(\frac{e^{\theta T}}{T} \int_{0}^{T} X_{t} d t-\frac{X_{T}}{2} e^{\theta T}\right) & =\lim _{T \rightarrow \infty}-\frac{e^{\theta T}}{2}\left(\frac{\mu}{\theta}\left(1-e^{-\theta t}\right)+e^{-\theta t} \int_{0}^{t} e^{\theta u} d L_{u}^{d}\right) \\
& =\frac{\mu}{2 \theta}-\lim _{T \rightarrow \infty} \frac{1}{2} \varphi_{T}=\frac{\mu}{2 \theta}-\frac{1}{2} \varphi_{\infty} .
\end{aligned}
$$

Using (2.5) and Young integral we have

$$
\begin{aligned}
\frac{e^{\theta T}}{T} \int_{0}^{T} X_{t}^{2} d t= & \frac{e^{\theta T}}{T} \int_{0}^{T}\left[\frac{\mu}{\theta}\left(1-e^{-\theta t}\right)+e^{-\theta t} \int_{0}^{t} e^{\theta u} d L_{u}^{d}\right]^{2} d t \\
= & \left(\frac{\mu}{\theta}\right)^{2}\left[e^{\theta T}\left(1-\frac{3}{2 \theta T}\right)-\frac{1}{2 \theta T} e^{-\theta T}+\frac{2}{\theta T}\right] \\
& +\frac{2 \mu \varphi_{T}}{T \theta^{2}}\left(e^{-\theta t}-1\right)+\frac{e^{\theta T}}{T} \int_{0}^{T} e^{-2 \theta t} \varphi_{t}^{2} d t
\end{aligned}
$$

and 


$$
\begin{aligned}
\frac{X_{T}}{2 T} e^{\theta T} \int_{0}^{T} X_{t} d t=\frac{1}{2 T} & {\left[\frac{\mu}{\theta}\left(1-e^{-\theta t}\right)+e^{-\theta t} \int_{0}^{t} e^{\theta u} d L_{u}^{d}\right] } \\
& \times e^{\theta T} \int_{0}^{T}\left[\frac{\mu}{\theta}\left(1-e^{-\theta t}\right)+e^{-\theta t} \int_{0}^{t} e^{\theta u} d L_{u}^{d}\right] \\
= & \frac{1}{2 T}\left(\frac{\mu}{\theta}-\frac{\mu}{\theta} e^{-\theta T}+e^{-\theta T} \varphi_{T}\right) \cdot\left(\frac{\mu}{\theta} e^{\theta T} T+\frac{\mu}{\theta^{2}}-\frac{\mu}{\theta^{2}} e^{\theta T}-\frac{\varphi_{T}}{\theta}\right) .
\end{aligned}
$$

Combiningleqref sec3-eq3.12 with \eqref sec3-eq3.13, we have

$$
\lim _{T \rightarrow \infty}=\frac{e^{\theta T}}{T} \int_{0}^{T} X_{t}^{2} d t-\frac{X_{T}}{2 T} e^{\theta T} \int_{0}^{T} X_{t} d t=\frac{1}{2}\left(\frac{\mu}{\theta}\right)^{2}-\frac{\mu}{2 \theta} \varphi_{\infty} .
$$

Finally, by (3.16) and (3.19) we can obtain the conclusion This completes the proof.

\section{Competing Interests}

The author declares that no competing interests exist.

\section{Funding Statement}

This work was supported by the This research is supported by the Distinguished Young Scholars Foundation of Anhui Province (1608085J06), the National Natural Science Foundation of China (11271020),Top talent project of university discipline(speciality) [gxbjZD03]

\section{References}

1. C. Bender, A. Lindner, M. Schicks. Finite variation of fractional Lévy processes. J. Theor. Probab. 25 (2012) 594612

2. J. P. Bishwal. Parameter estimation in stochastic differential equations. Berlin: Springer. 24 (2008).

3. F. Comte, E. Renault. Long memory in continuous-time stochastic volatility models. J. Econometr. 8 (1998) 291-323.

4. F. Comte, L. Coutin and E. Renault, Affine fractional stochastic volatility models. Ann Finance 8(23) (2012) 337-378.

5. S. Corlay, J. Lebovits and J. L. Véhel, Multifractional stochastic volatility models. J. Econometr. 24(2) (2014) 364-402.

6. H. M. Dietz, Y. A. Kutoyants. Parameter estimation for some non-recurrent solutions of SDE. Statist. Decisions. 21 (2003) 29-46.

7. H. Fink, C. Klüppelberg. Fractional Lévy-driven Ornstein-Uhlenbeck processes and stochastic differential equations. Bernoulli. 17 (2011) 484-506.

8. Y. $\mathrm{Hu}, \mathrm{D}$. Nualart. Parameter estimation for fractional Ornstein-Uhlenbeck process. Statist. Probab. Lett. 80 (2010) 1030-1038.

9. Y. A. Kutoyants. Statistical Inference for Ergodic Diffusion Processes. Berlin, Heidelberg: Springer, 2004. 
10. T. Marquardt. Fractional Lévy processes with an application to long memory moving average processes. Bernoulli 12(6) (2006) 1099-1126.

11. B. L. S. Prakasa Rao. Parametric estimation for linear stochastic delay differential equations driven by fractional Brownian motion. Random Oper. Stochastic Equations. 16 (2008) 27-38.

12. G. Samorodnitsky and M. Taqqu. Stable Non-Gaussian Random processes: Stochastic Models with Infinite Varice. Champman \& Hall, New York. 1994.

13. K. Sato. Lévy Processes and Infinitely Divisible Distributions . Cambridge: Cambridge University Press, 1999.

14. H. Tikanmäki, Y. Mishura. Fractional Lévy processes as a result of compact interval integral transformation. Stoch. Anal. Appl. 29 (2011) 1081-1101.

15. O. Vasicek. An equilibrium characterization of the term structure. J. Financial Ecori. 5(2)(1977) 177-188.

16. W. Xiao, J. Yu. Asymptotic theory for estimating the persistent parameter in the fractional vasicek model. Singapore Management University, School of Economics, Paper No. 9-2016. 